\title{
Assessment of Ocriplasmin Effects on the Vitreoretinal Compartment in Porcine and Human Model Systems
}

\author{
Bart Jonckx, Michael Porcu, Aurelie Candi, Isabelle Etienne, Philippe Barbeaux, and \\ Jean H. M. Feyen
}

ThromboGenics NV, Gaston Geenslaan 1, 3001 Leuven, Belgium

Correspondence should be addressed to Bart Jonckx; bart.jonckx@thrombogenics.com

Received 27 April 2017; Accepted 17 September 2017; Published 29 October 2017

Academic Editor: Taras Ardan

Copyright (c) 2017 Bart Jonckx et al. This is an open access article distributed under the Creative Commons Attribution License, which permits unrestricted use, distribution, and reproduction in any medium, provided the original work is properly cited.

\begin{abstract}
Ocriplasmin (Jetrea ${ }^{\circledR}$ ) is a recombinant protease used to treat vitreomacular traction. To gain insight into vitreoretinal observations reported after ocriplasmin treatment, we have developed an in vivo porcine ocriplasmin-induced posterior vitreous detachment (PVD) model in which we investigated vitreoretinal tissues by optical coherence tomography, histology, and cytokine profiling. Eight weeks postinjection, ocriplasmin yielded PVD in $82 \%$ of eyes. Subretinal fluid (85\%) and vitreous hyperreflective spots (45\%) were resolved by week 3. Histological analysis of extracellular matrix (ECM) proteins such as laminin, fibronectin, and collagen IV indicated no retinal ocriplasmin-induced ECM distribution changes. Retinal morphology was unaffected in all eyes. Cytokine profiles of ocriplasmin-treated eyes were not different from vehicle. In cell-based electrical resistance assays, bloodretinal barrier permeability was altered by ocriplasmin concentrations of $6 \mu \mathrm{g} / \mathrm{mL}$ and higher, with all effects being nontoxic, cell-type specific, and reversible. Ocriplasmin was actively taken up by RPE and Müller cells, and our data suggest both lysosomal and transcellular clearance routes for ocriplasmin. In conclusion, transient hyperreflective spots and fluid in a porcine ocriplasmin-induced PVD model did not correlate with retinal ECM rearrangement nor inflammation. Reversible in vitro effects on blood-retinal barrier permeability provide grounds for a hypothesis on the mechanisms behind transient subretinal fluid observed in ocriplasmin-treated patients.
\end{abstract}

\section{Introduction}

Posterior vitreous detachment (PVD), the release of the vitreous cortex from the internal limiting membrane, is a normal physiologic process related to the aging eye. Incomplete PVD can lead to vitreomacular traction (VMT), which may in turn cause retinal damage and loss of visual acuity [1]. One option to treat VMT is vitrectomy surgery, in which the vitreous is removed from the eye and the traction is surgically resolved. Although effective, vitrectomy surgery is invasive and has a known risk profile with potential retinal damage and cataract formation [2, 3]. A novel nonsurgical intervention for VMT is intravitreal injection of ocriplasmin (trade name Jetrea) [4]. Ocriplasmin is a truncated form of plasmin that is administered via intravitreal injection to induce PVD. It is proposed that the PVD results from enzymatic cleavage of extracellular matrix proteins contributing to the traction, such as fibronectin and laminins at the inner limiting membrane [5-7]. Ocriplasmin, which was approved by the FDA in 2012 and EMA by 2013, has been shown to be efficacious for treatment of VMT with or without fullthickness macular hole $(<400 \mu \mathrm{m})$ in several clinical studies $[4,6,8,9]$. Appropriate patient selection, for example, absence of epiretinal membranes, results in a substantially increased efficacy $[8,10-12]$. The recent OASIS study evaluating the efficacy of ocriplasmin in VMT showed that $41.7 \%$ of patients achieved resolution at day 28 versus $6.2 \%$ in sham [13].

Although ocriplasmin shows promise as a less invasive alternative treatment for patients with VMT, vitreoretinal changes, such as subretinal fluid, vitreous hyperreflective spots, ellipsoid zone attenuation, and ERG profile changes, have been observed in a subset of patients [13]. Most of the retinal adverse events in human eyes have been reported to be transient or reversible over time, typically within 2 months after injection [4, 12, 14-18]. Even cases of severe visual 
acuity impairment and visual field loss have been reported to resolve quite completely over extended periods of 4 months to 36 months $[19,20]$. Recently, the 24 -month, prospective, randomized, double-masked, sham-controlled OASIS trial demonstrated the long-term efficacy and safety of ocriplasmin, demonstrating improved resolution of symptomatic VMA compared with previous phase 3 trials with no new safety signals identified $[13,21]$. Furthermore, ocriplasmin has been shown to produce clinically meaningful visual function benefits and ERG reductions were shown to correlate with superior visual improvement by the end of the study [22-24].

To understand the vitreoretinal changes observed in a subset of ocriplasmin-treated eyes, further molecular and functional insight into the impact of ocriplasmin on the vitreoretinal compartment are required. Here, we report insights obtained by studying vitreoretinal tissues from an ocriplasmin-treated porcine PVD model as well as retinal cell models.

\section{Methods}

2.1. Pig Posterior Vitreous Detachment (PVD) Model. All animals were housed and cared for in compliance with the FELASA guidelines and recommendations. The study was approved by the Ethics Committee for Animal Research at Medanex Clinic (EC MxCl-2014-028) and conformed to the ARVO statement for the use of animals in ophthalmic and vision research. Male farm pigs (Sus scrofa domesticus) were anesthetized and positioned on a surgical table, and $10 \mathrm{~mL}$ retrobulbar anesthetic (xylazine 1\%) was injected. $100 \mu \mathrm{L}$ ocriplasmin $(96 \mu \mathrm{g})$ or vehicle $(5 \mathrm{mM}$ sodium citrate in $0.9 \%$ sodium chloride, $\mathrm{pH} 3.1$ ) was injected midvitreally. $96 \mu \mathrm{g}$ ocriplasmin in $3.3 \mathrm{~mL}$ porcine vitreous corresponds to the clinical dose of $125 \mu \mathrm{g}$ ocriplasmin administered intravitreally to $4.5 \mathrm{~mL}$ human vitreous. Follow-up was performed by weekly spectral domain optical coherence tomography (OCT) using a Heidelberg Spectralis device. Two studies were performed. In the first study, 13 animals were followed up 8 weeks by OCT. Two animals were excluded from analysis due to events not related to drug administration. In the second study, 21 animals were followed by OCT and sacrificed at regular time points after injection for histology and cytokine profiling (4 animals sacrificed in week 1, 5 animals in week 2, 4 animals in week 4 , and 8 animals in week 6).

2.2. Histology and Vitreous Cytokine Profiling. Eyes were fixed for 48 hours in $4 \%(w / v)$ paraformaldehyde solution, rinsed in PBS, and stored overnight in 3\% $(w / v)$ sucrose in PBS. Deparaffinized sections of $7 \mu \mathrm{m}$ thickness were stained with either hematoxylin and eosin or antibodies specific for pan-laminin (NB600-680, Novus), fibronectin (ab6584, Abcam), collagen IV (1340-08, Sanbio), or Iba-1 (019-19741, Wako). Vitreous detection of CCL-2 was performed using a custom Mesoscale Discovery ELISA assay. Detection of the remaining cytokines was performed using MILLIPLEX ${ }^{\circledR}$ MAP technology (Millipore). Four untreated eyes as well as two lipopolysaccharides treated eyes (100 ng/eye) were included as controls [25]. Statistical differences were evaluated using the Mann-Whitney $U$ test.

2.3. Transepithelial/Transendothelial Electrical Resistance (TEER) Measurements. Primary human retinal microvascular endothelial cells (HRMEC, Cell Systems) were cultured in EndoGRO-MV-VEGF Media (Millipore). Human retinal pigment epithelium cells (ARPE-19, ATCC) were cultured in DMEM supplemented with 10\% FBS according to supplier instructions. In brief, cells were seeded in 24-well transparent $0.4 \mu \mathrm{m}$ pore PET culture inserts (Greiner). Inserts were placed in the cellZscope 24-well module (NanoAnalytics) and TEER and capacitance values were recorded in one hour intervals. Once at TEER plateau, treatment in serum-free media was added to the upper transwell compartment. Following treatment, washout was performed to investigate recovery. Data were analyzed in cellZscope software and GraphPad Prism.

2.4. Alexa 488-Labeled Ocriplasmin Experiments. Ocriplasmin was fluorescently labeled using an Alexa 488 protein labeling kit (Thermo Fisher). Inactive ocriplasmin was generated either by autolysis for $24 \mathrm{~h}$ or by addition of the inhibitor Val-Phe-Lys. ARPE-19 cells or freshly isolated porcine Müller cells, cultured in DMEM with 10\% FBS, were treated with a solution of $10 \mu \mathrm{g} / \mathrm{mL}$ ocriplasmin in HBSS. As vehicle, $5 \mathrm{mM}$ sodium citrate in $0.9 \% \mathrm{NaCl}$ ( $\mathrm{pH} 3.1)$ was used. For immunofluorescence, cells were grown on 8-well-cultured slides. After treatment, cells were washed, fixed in 4\% PFA, and stained with indicated antibodies: Rab7 clone D95F2, Rab11 clone D4F5 (Cell Signaling Technologies), and goat anti-rabbit Alexa 555 (Life technologies) within 48 h. Cells were imaged via epifluorescence (Zeiss Axio Imager 2) as well as confocal microscopy (Nikon $\mathrm{C} 2+$ ). For flow cytometry, cells were washed with PBS after treatment and detached with Accutase (Sigma-Aldrich). Data were acquired and analyzed on a FACSCanto (BD Biosciences) using the FACSDiva software II. Additional information can be found in supplemental methods available online at https://doi.org/ $10.1155 / 2017 / 2060765$.

\section{Results}

3.1. Posterior Vitreous Detachment (PVD) and Transient Retinal Observations in the Porcine PVD Model. In order to model ocri plasmin-induced PVD, farm pigs were injected with $96 \mu \mathrm{g}$ ocriplasmin or vehicle (to the contralateral eye) midvitreally and followed up by OCT. In ocriplasmintreated eyes, PVD was observed from week 2 onwards. After 8 weeks, PVD was observed in $82 \%(9 / 11)$ of ocriplasmin eyes, compared to $9 \%(1 / 11)$ of vehicle eyes (Figure 1(a), Supplemental Table 1). PVD was identified on OCT images as a hyperreflective interface in the vitreous (Figures 1(b), 1(c), 1(d), and 1(e)). All PVDs observed in porcine eyes were partial. Analysis of OCT images did not reveal significant layer changes when compared to control eyes. However, subretinal fluid (SRF) was observed after ocriplasmin administration, reaching a maximum of $85 \%(11 / 13)$ one week after 


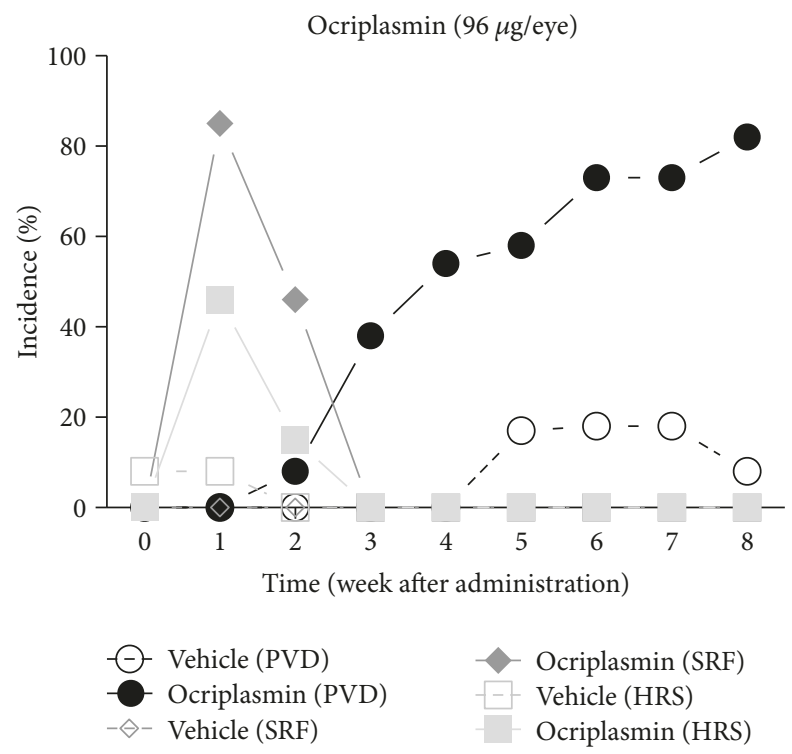

(a)

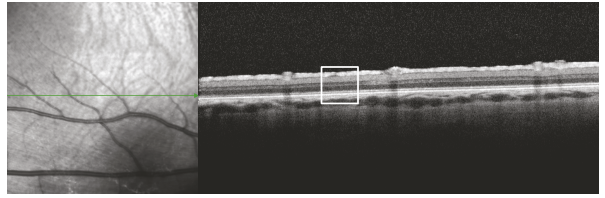

(b)

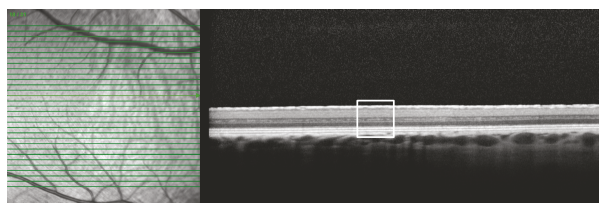

(d)
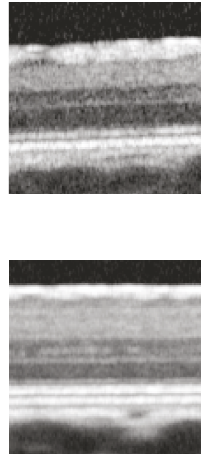

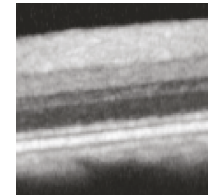

(c)
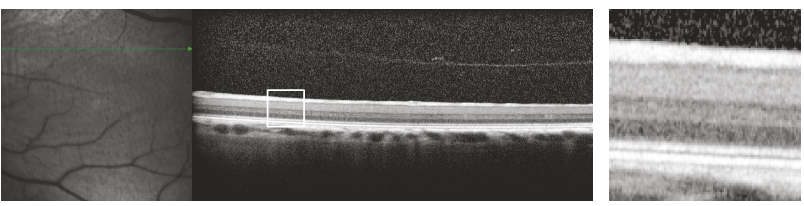

(e)

FIGURE 1: Optical coherence tomography follow-up after ocriplasmin versus vehicle in the porcine PVD model. (a) Incidence of PVD, subretinal fluid (SRF), and hyperreflective spots (HRS) after administration of vehicle or $96 \mu \mathrm{g} /$ eye ocriplasmin ( $n=13$ eyes per group). (b-e) Representative OCT images of the retina 5 weeks after treatment with either vehicle (b, d) or ocriplasmin (c, e) in a locationmatched retinal region where no PVD was present $(b, c)$ or where PVD was observed (d, e). Left images represent fundus image, middle image shows OCT of the retina, and white inset boxes indicate locations of OCT detail (right images). No disruption of retinal layers is observed.

administration, which diminished to $46 \%(6 / 13)$ in week 2 and disappeared completely from week 3 onwards (Figures 1(a) and 2). The severity of SRF was limited with an average volume of $0.062 \pm 0.012 \mathrm{~mm}^{3}$ (average \pm SEM; range: $0.00085-0.149 \mathrm{~mm}^{3}$ ) one week after administration and diminishing thereafter (Figure 2(c)). SRF was not observed in vehicle eyes. Hyperreflective spots (HRS) in the vitreous were observed in both groups (Supplemental Figure 1), reaching a maximum of $46 \%(6 / 13)$ in ocriplasmin-treated eyes versus $8 \%(1 / 13)$ in vehicle-treated eyes. All HRS were resolved by week 3 . No changes were observed in the region of the ellipsoid zone.

\subsection{Retinal Morphology and Extracellular Matrix} Distribution in the Porcine PVD Model. In a follow-up study, farm pigs were treated and followed by OCT as described above. However, animals were sacrificed and vitreoretinal tissues were collected at specific time points up to 6-weeks postinjection. Periodic acid-Schiff (PAS) staining confirmed the presence of ocriplasmin-induced PVD as well as the absence of morphology changes in the retina and retinal layers as observed by OCT (Figure 3 ). We investigated the vitreoretinal distribution of laminin, fibronectin, and collagen IV, which are preferential extracellular matrix (ECM) substrates of ocriplasmin by immunohistochemistry. In line with its hypothesized mode of action, ocriplasmin-induced PVD segregated the inner limiting membrane staining into 2 layers. One layer remained attached to the retinal surface, while the other layer migrated with the PVD interface into the vitreous. In the retina itself, laminin, fibronectin, and collagen IV distribution remained localized to the blood vessels and were comparable to the vehicle at 2 or 5 weeks after ocriplasmin treatment (Figure 3).

3.3. Characterization of Inflammatory Markers in the Porcine PVD Model. Staining for Iba-1, a retinal microglia and 


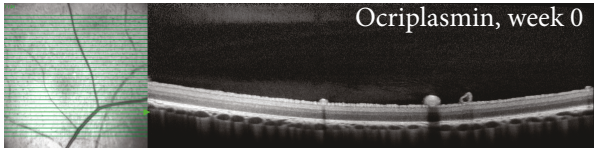

(a)

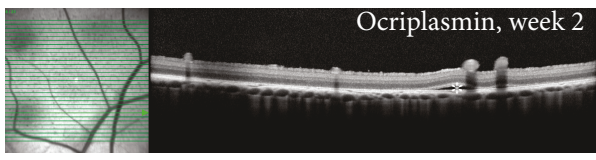

(c)

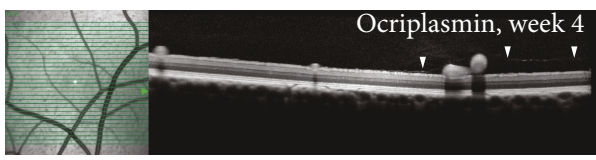

(e)

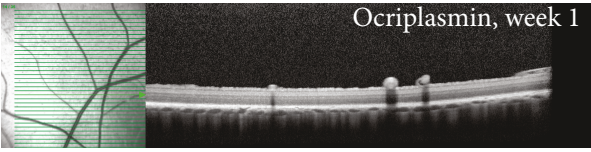

(b)

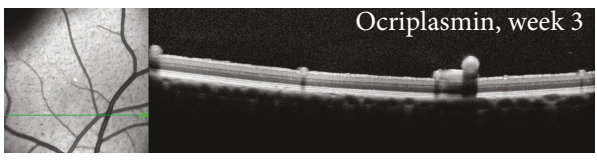

(d)

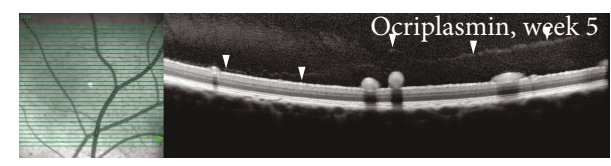

(f)

FIGURE 2: Optical coherence tomography time series follow-up after ocriplasmin in the porcine PVD model. (a-f) OCT follow up of a representative animal over time. A location-matched OCT is shown over a period of 5 weeks, from before injection until after PVD induction. SRF appears in week 2 (c) disappears by week 3 (d). PVD is observed in week 4 (e) and continues to enlarge in week 5 (f). For clarity, the inner retinal vessels in pigs are located more superficial on top of the inner limiting membrane; underneath these blood vessels, a prominent "shadow" can be observed in the OCT image caused by the opacity of the red blood cells in these vessels.

macrophage marker, indicated an increase of Iba- $1^{+}$cells in retinas of ocriplasmin-treated eyes over vehicle eyes (Figure 4). Although a statistically significant difference in $\mathrm{Iba}^{+}$cells remained present at week 6 postinjection, the number of $\mathrm{Iba1}^{+}$cells showed a downward trend over time. Compared to the level of $\mathrm{Iba}^{+}$cells obtained after an LPS challenge, ocriplasmin-induced changes, although significant, were minimal and close to the range of naive eyes.

In addition, we determined the levels of 14 inflammatory markers in vitreous samples originating from the PVD model: CCL-2, GM-CSF, IFN- $\gamma$, TNF- $\alpha$, IL- $1 \alpha, 1 \beta, 2,4,6$, $8,10,12,18$, and IL-1Ra (Figure 5, Supplemental Table 2). Overall, vitreous levels of these cytokines remained in the range of noninjected eyes in all treatment groups except for the LPS-treated control group, where eyes showed a significant inflammatory response. No statistically significant differences were found between cytokine levels in vehicle versus ocriplasmin-treated eyes. Neither could we detect statistically significant differences between both groups when we restricted the analysis to either early (week 1 and 2) or late (week 4 and 6) time points after injection. Irrespective of treatment, we detected a modest but transient increase in several cytokines $(p<0.05$ for IL-1Ra, IL-1 $\beta$, CCL-2, IL-8, 12,18 and GM-CSF, see Figure 5) at early postinjection time points. Also, it has to be noted that in two porcine eyes some late increases in levels of IFN- $\gamma$, TNF- $\alpha$, IL-2, and IL- 4 were observed 6 weeks after treatment with ocriplasmin. Histological analysis of these eyes did not indicate any changes in retinal morphology or $\mathrm{Iba}^{+}$cell count.

\subsection{Ocriplasmin Effects on Cell-Based Blood-Retinal Barrier} Models. To evaluate the effect of ocriplasmin on retinal barrier integrity and thus the potential of ocriplasmin to create imbalances in retinal fluid homeostasis, we measured transendothelial or transepithelial electrical resistance (TEER) in monolayer cultures of human microvascular endothelial cells (HRMEC) and human retinal pigment epithelium cells (ARPE-19). TEER values reflect the integrity of tight junctions. Cell monolayers were allowed to form tight junctions and were subsequently treated with a concentration range of ocriplasmin, vehicle, or inactive (autolyzed) ocriplasmin. For HRMEC cells, ocriplasmin concentrations below $6 \mu \mathrm{g} / \mathrm{mL}$ had no effects on TEER. Ocriplasmin concentrations of 6 and $12 \mu \mathrm{g} / \mathrm{mL}$ decreased TEER values in a concentration dependent manner (Figure 6). This effect was reversible, with TEER recovering back to vehicle level within 36 hours. Next, we assessed the effect of ocriplasmin on ARPE-19 monolayers. In contrast to endothelial monolayers, ocriplasmin treatment of RPE cells yielded a small increase in TEER (Figure 6). This increase was transient or reversible after treatment washout. In both cell types, inactive ocriplasmin did not affect TEER. No observations were made indicating cell toxicity, such as increases in the capacitance profiles due to changes in cellular adherence or cell death (data not shown).

\subsection{Retinal Cells Internalize Ocriplasmin in Active Transport} Organelles. We investigated whether retinal cells could participate in the uptake and clearance of ocriplasmin. Human retinal pigment epithelium cells (ARPE19) and primary porcine Müller cells were treated with Alexa 488 fluorescently labeled ocriplasmin, and localization was evaluated by fluorescence microscopy. Thirty minutes after treatment, microscopy indicated the presence of labeled ocriplasmin inside both cultured Müller and RPE cells (Figure 7(a)). Interestingly, A488-ocriplasmin signal was present in foci, which were not detected after treatment with label alone or inactive ocriplasmin. Indeed, flow cytometry data confirmed that enzymatically inactive forms of ocriplasmin were taken up at a much slower rate than the active form (Figure $7(b)$ ). The focal intracellular distribution of labeled ocriplasmin 
Vehicle

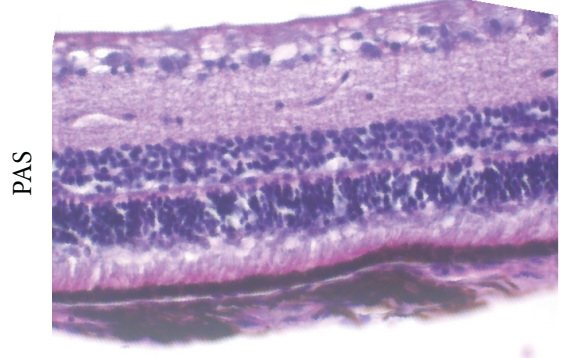

(a)

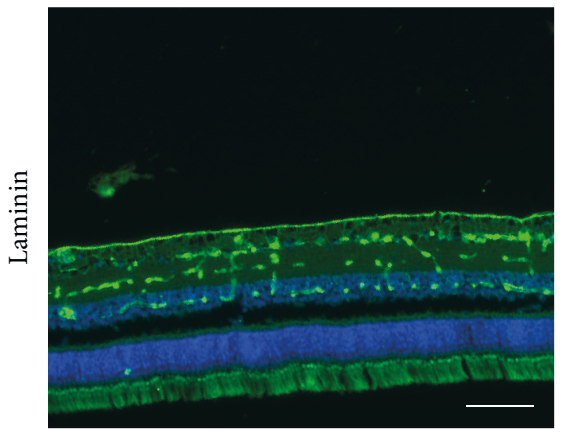

(e)

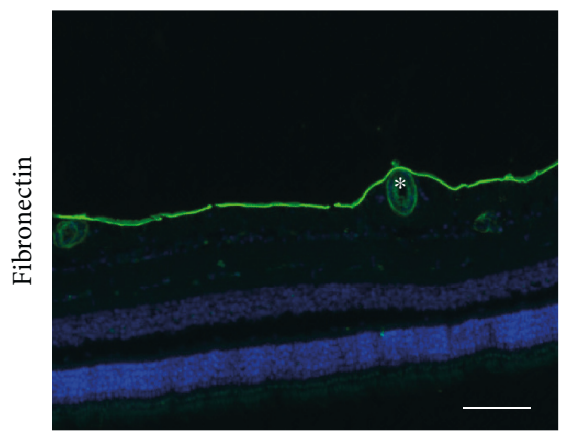

(h)

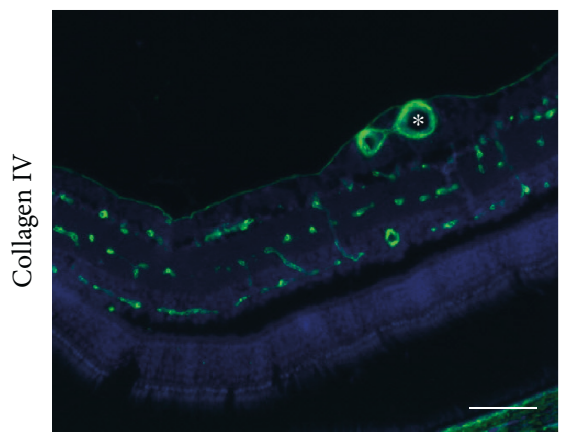

(k)
Ocriplasmin, week 2

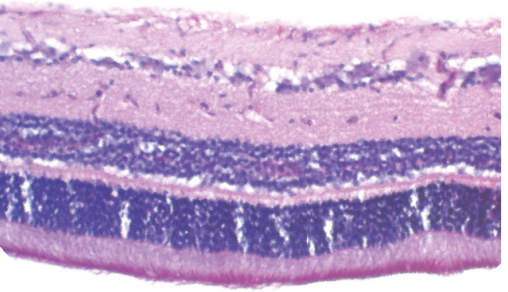

(b)

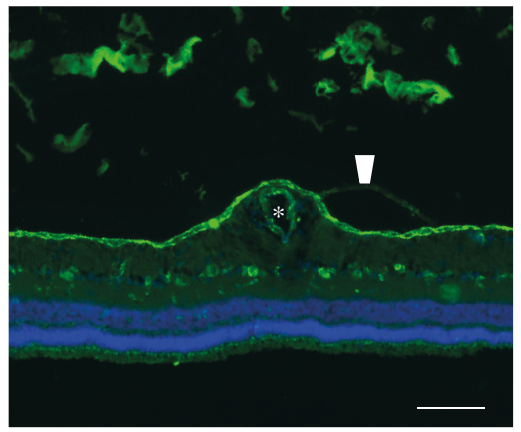

(f)

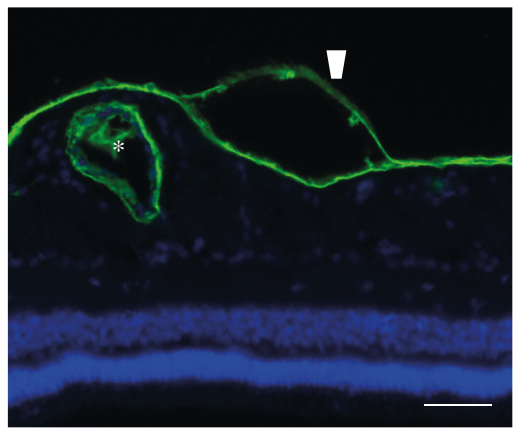

(i)

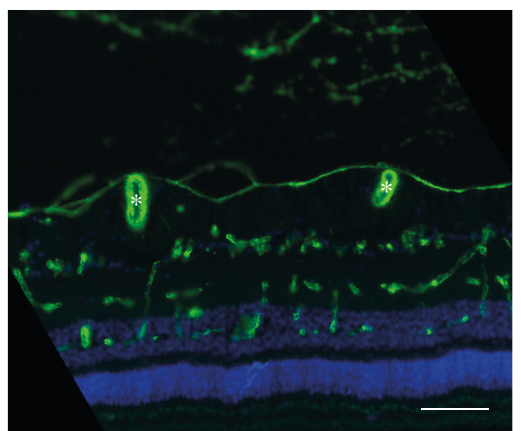

(1)
Ocriplasmin, week 5

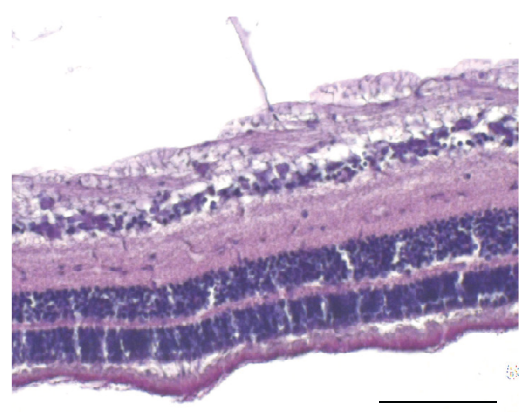

(c)

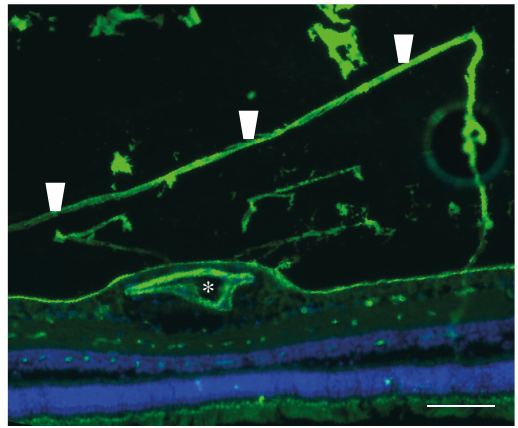

(g)

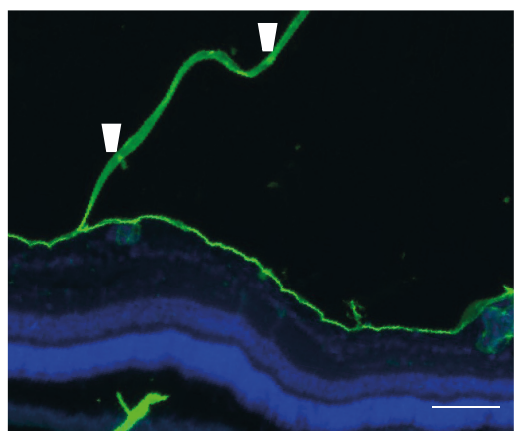

(j)

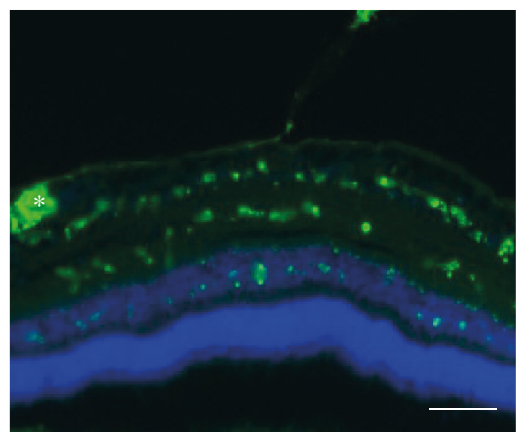

(m)

FIgURE 3: Effect of ocriplasmin on vitreoretinal architecture and extracellular matrix distribution in the porcine PVD model. (a-l) Representative histology images from porcine retinas 2 and 5 weeks after administration of vehicle or ocriplasmin. (a, b, and c: PAS; d, e, and f: laminin; g, h, and i: fibronectin; j, k, and l: collagen IV; d-l: specific stain in green, nuclei in blue). Scale bar: $100 \mu \mathrm{m}$. ${ }^{*}$ Large blood vessels which are situated superficially in the ILM in the pig. Arrowhead indicates ILM separation/PVD.

suggested that it could be incorporated into vesicles. Using confocal microscopy, we investigated whether ocriplasmin was present in endosomes. In both Müller and RPE cells, a portion of ocriplasmin foci was found to colocalize with Rab7 positive late endosomes, which are committed to become lysosomes. In contrast, in RPE cells, we could also 


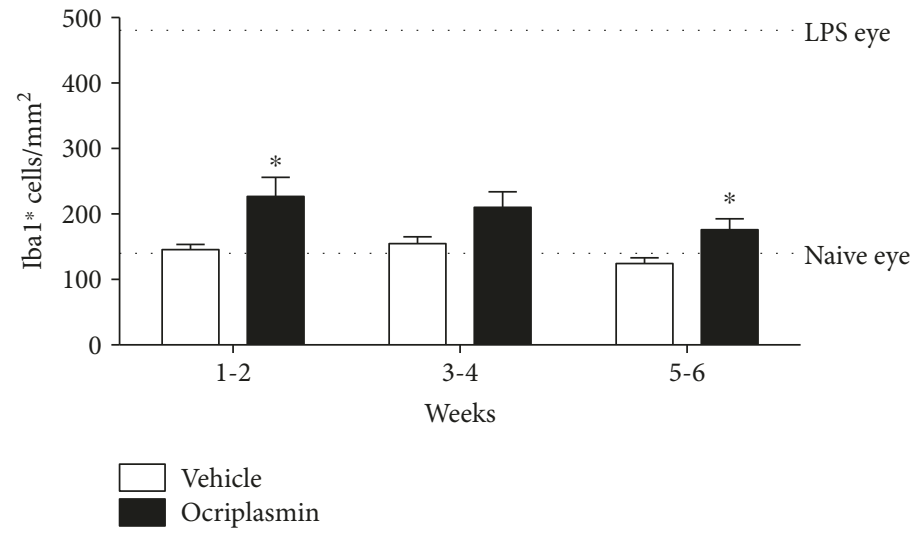

(a)

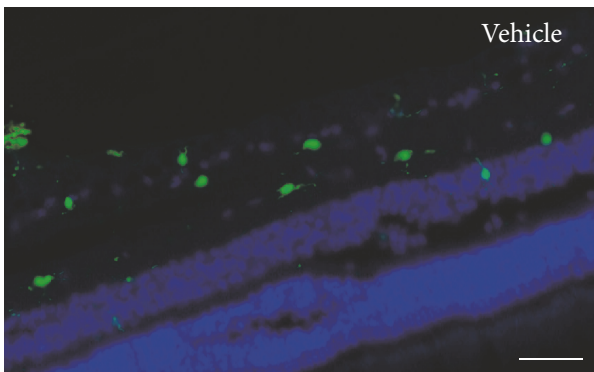

(b)

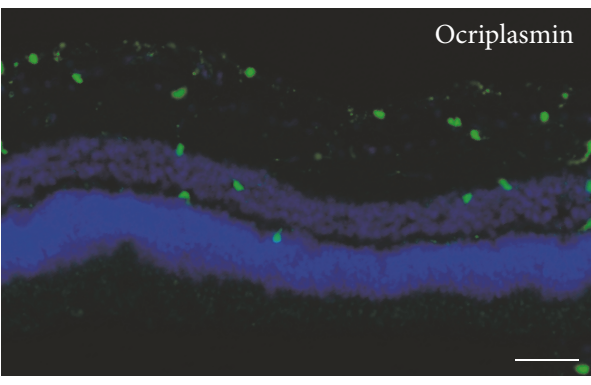

(c)

FIgURE 4: Staining for the inflammatory marker Iba1 in the porcine PVD model. (a) Quantification of Iba1 ${ }^{+}$cells in the retina after treatment with either vehicle or ocriplasmin. Samples from 4 animals sacrificed in week 1, 5 animals in week 2, 4 animals in week 4, and 8 animals in week 6. ${ }^{*} p<0.05$ versus time-matched vehicle control (Student's $t$-test). (b, c) Representative images of the Iba1 ${ }^{+}$stain 1 week after vehicle (b) or ocriplasmin (c) treatment. Scale bar: $100 \mu \mathrm{m}$.

detect ocriplasmin in Rab11 positive endosomes, which recycle to the plasma membrane (Figure $7(\mathrm{c})$ ).

\section{Discussion}

In order to better understand some of the transient vitreoretinal observations made in a subset of ocriplasmin-treated patients, we developed and analyzed vitreoretinal tissues from a porcine ocriplasmin-induced posterior vitreous detachment (PVD) model. We chose the porcine eye as it is a highly relevant and suitable model to investigate human eye diseases such as glaucoma [26], proliferative vitreoretinopathy $[27,28]$, and retinitis pigmentosa $[29,30]$. The porcine eye and retina share many similarities with that of the human eye [31-36], resembling the human retina in number and distribution of rods and cones, shape, vasculature, and function [37-42]. Porcine vitreous is similar to human vitreous in terms of volume and biochemical composition [43]. Additionally, the porcine eye is an attractive nonprimate model for exploring preclinical efficacy and safety of novel surgical and pharmaceutical therapies [44-46]. The mode of delivery of ocriplasmin in the pig's eye is highly comparable to intravitreal injection in the human eye; midvitreal placement of the injection yields similar distances to the retina and lens, in contrast to other species such as the monkey or rodent.
When injected with ocriplasmin at a similar concentration and injection volume compared to human, ocriplasmininduced PVD in the porcine model occurs from week 2 postinjection onward. At 8-weeks postinjection, PVD was observed in $82 \%$ of ocriplasmin-treated pigs as compared to $9 \%$ of vehicle-treated eyes. Further investigation of vitreoretinal morphology by OCT indicated no changes in retinal morphology during the 8-weeks postinjection follow-up period but did indicate the occurrence of transient vitreoretinal effects, such as vitreal hyperreflective spots and subretinal fluid after ocriplasmin treatment. These changes occurred rapidly after injection (peak incidence at week 1) and were transient in nature, resolving by week 3 . These observations correlate with observations made in patients and provided the opportunity to study the underlying biological processes more in detail. Ellipsoid zone changes, on the other hand, were not observed in the pig model. It remains unclear if this is a limitation of the model due to the species differences. In this respect, it should be noted that the ellipsoid zone is less distinct in the pig compared to human.

It has been hypothesized that transient retinal observations induced by ocriplasmin are the result of partial or total removal of ECM inside the retina due to enzymatic cleavage [47]. Our results indicate that treatment with $29 \mu \mathrm{g} / \mathrm{mL}$ ocriplasmin $(96 \mu \mathrm{g} / \mathrm{eye}$, pig vitreous volume $=3.3 \mathrm{~mL}[48])$ has no clear impact on the retinal staining of both laminin, 

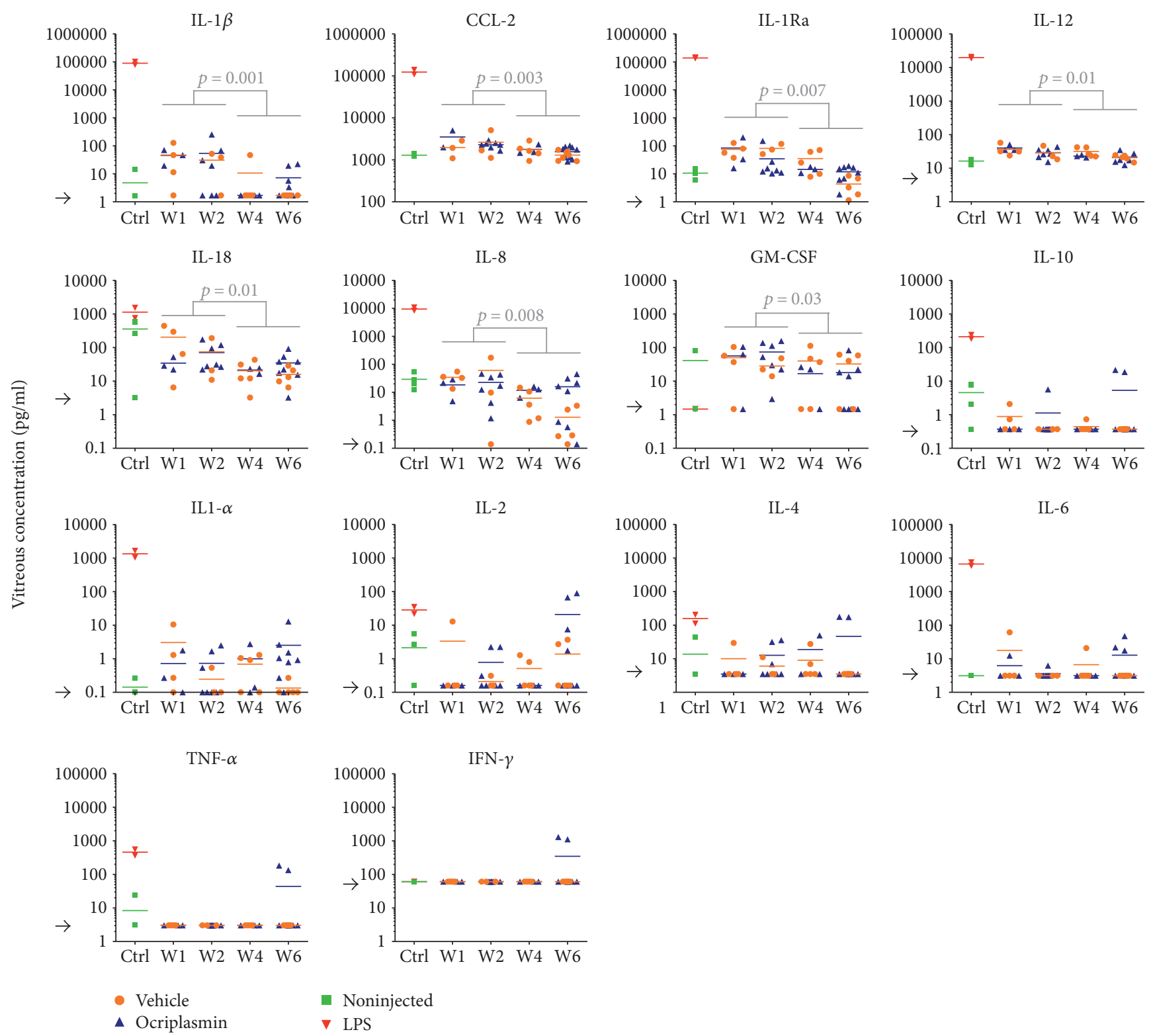

FIGURE 5: Cytokine profile of porcine vitreous samples. Vitreous of porcine eyes was collected at the time of sacrifice, either at week (w) 1, 2, 4, or 6 after treatment. Arrowheads represent assay detection limits (detection limit for CCL-2 not indicated as lower than range of the graph). Horizontal lines represent sample means. $P$ values were calculated using Mann-Whitney $U$ test.

fibronectin, and collagen IV, except locally at the inner limiting membrane, in agreement with the proposed mechanism of action of ocriplasmin. In knockout models of ECM such as laminin $\beta 2$ and $\gamma 3$, retinal and ERG changes have been observed $[49,50]$. As we observe the largely unchanged retinal distribution of ECM proteins, we hypothesize that it is unlikely that ocriplasmin induces such an exaggerated pharmacological response as observed in conditions of total ECM protein deficiency. In addition, retinal cell types such as endothelial cells, Müller cells, and retinal pigment epithelium cells have been described to produce these proteins, thereby regenerating the ECM [51-53].

The transient occurrence of SRF in the porcine PVD model was restricted to ocriplasmin-treated eyes, and transient vitreous HRS occurred more frequently in ocriplasmin-treated eyes. By examining vitreoretinal tissues from our porcine PVD model, we investigated if these observations resulted from an acute inflammatory response. While histological analysis of the porcine retinas used in our study did indicate a minor increase of Iba1-positive cells in ocriplasmin-treated eyes, levels remain in the range of healthy eyes [54]. Additionally, cytokine profiling indicated no significant difference between vehicle- and ocriplasmintreated eyes. A minor and transient inflammatory response was observed in all eyes postinjection, irrespective of treatment and indicative of an effect related to the injection procedure. Finally, for a number of cytokines, levels increased at late time points in 2 ocriplasmin-treated eyes 

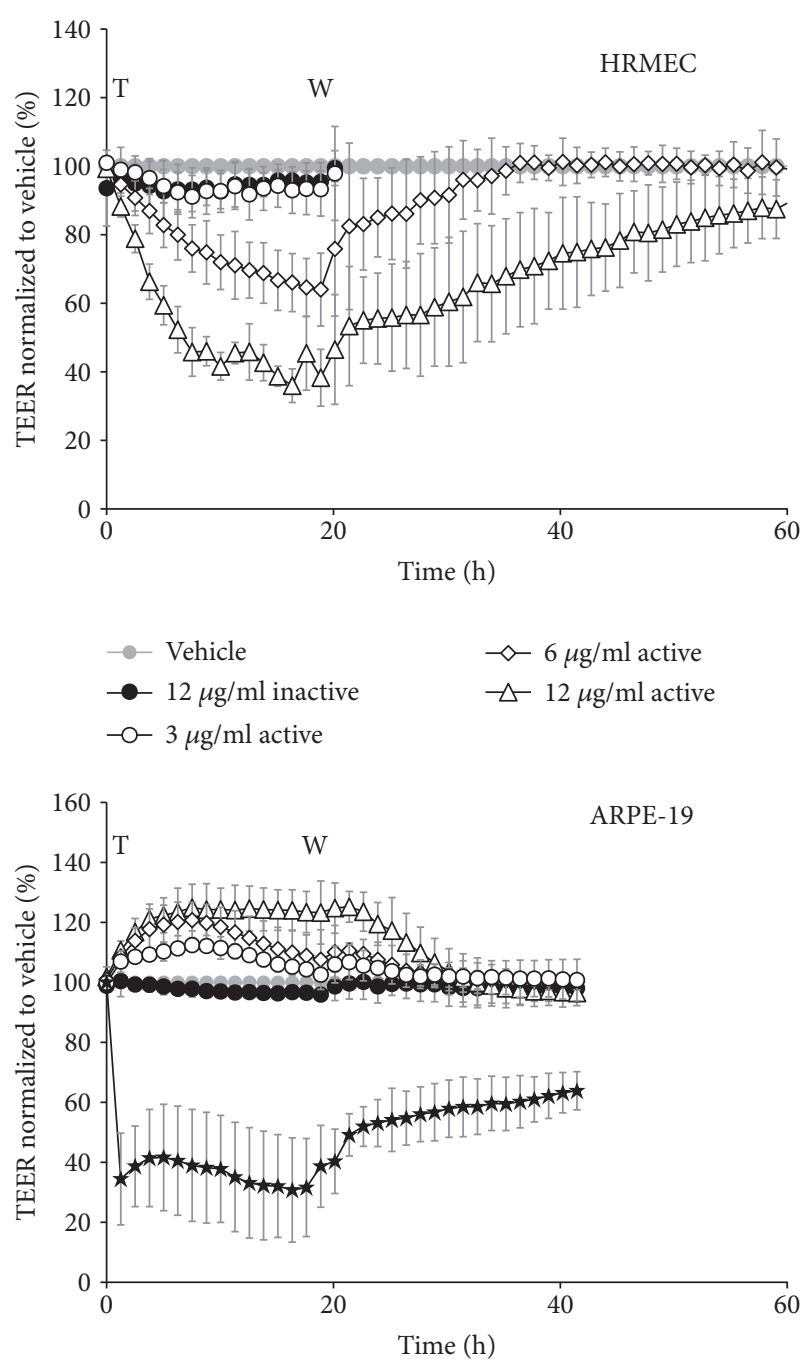

$\begin{array}{ll}- \text { Vehicle } & \checkmark 6 \mu \mathrm{g} / \mathrm{ml} \text { active } \\ --12 \mu \mathrm{g} / \mathrm{ml} \text { inactive } & -12 \mu \mathrm{g} / \mathrm{ml} \text { active } \\ -0-3 \mu \mathrm{g} / \mathrm{ml} \text { active } & \rightarrow 2 \mathrm{mM} \text { EGTA }\end{array}$

FIgURE 6: Effect of ocriplasmin treatment on TEER. Transendothelial or -epithelial resistance of HRMEC and ARPE19 monolayer cultures was monitored in function of time after applying the indicated treatments. Values are expressed as mean of biological triplicates \pm SD. T: treatment; W: washout.

but were not correlated to morphological changes. These results indicate that ocriplasmin-induced PVD, SRF, and HRS do not appear to correlate with inflammatory pathways.

An alternative potential mechanism underlying the emergence of SRF is that ocriplasmin could affect retinal fluid homeostasis. Investigation in in vitro models of the inner (HRMEC) and outer (ARPE-19) blood-retinal barrier indicated that ocriplasmin could modulate barrier permeability in a cell- and concentration-specific manner. All effects were reversible and no signs of cellular toxicity were observed. In HRMEC, concentrations starting at $6 \mu \mathrm{g} / \mathrm{mL}$ ocriplasmin reduced monolayer TEER, whereas lower concentrations had no effect. In contrast, ocriplasmin slightly increased TEER of RPE monolayers. Our observations of ocriplasmin-induced TEER increase seem remarkable but have been reported previously for other proteases $[55,56]$. Unfortunately, no good hypotheses exist on the in vivo relevance of this mechanism, but such imbalance could result in fluid accumulation. The RPE is known to keep the subretinal space "dry" by maintaining appropriate fluid homeostasis $[57,58]$. It remains to be confirmed whether the observed in vitro TEER changes are physiologically significant, since ocriplasmin is rapidly inactivated after injection due to autolysis and endogenous circulating inhibitors, making the local concentration of proteolytically active ocriplasmin difficult to estimate. Furthermore, alternative mechanisms could lead to SRF accumulation in vivo. As a result of its proteolytic activity, ocriplasmin could, for instance, induce accumulation of proteins or protein fragments that increase the osmotic pressure at the subretinal space, hereby favoring water retention in the subretinal space [57]. Finally, it cannot be excluded that part of the mode of action is mediated by proteases operating downstream of ocriplasmin (e.g., MMPs) [59]. Further experiments are required to investigate the contribution of these pathways to fluid homeostasis.

Finally, retinal cells, such as Müller glia and retinal pigmented epithelium (RPE) cells have been shown to participate in retinal uptake, transport and clearance of proteins, and intravitreally administered therapeutics, such as antiVEGFs [60-63]. We have investigated whether this could also be the case for ocriplasmin. Our data indicate that both Müller and RPE cells internalize ocriplasmin, and at least a part of ocriplasmin seems to be internalized via active endocytosis. Although the specific uptake pathway remains unclear, the different uptake dynamics of active versus inactive ocriplasmin uptake does indicate a mechanism dependent on the enzymatic activity. The presence of ocriplasmin in Rab7 positive endosomes suggests that Müller and RPE cells are involved in clearance of ocriplasmin by directing at least part of internalized ocriplasmin to a lysosomal degradation fate [64]. In RPE cells specifically, ocriplasmin signal is also present in Rab11 positive recycling endosomes. Although we did not evaluate the directionality of ocriplasmin transport, RPE-mediated directional transport of therapeutic antibodies, such as anti-VEGFs, towards the choroid has been described, suggesting an additional route of ocriplasmin clearance from the eye $[61,63]$. More study is required to evaluate whether internalized ocriplasmin maintains proteolytic activity as well as to elucidate the fate of ocriplasmin after intravitreal injection in ex vivo or in vivo models, where the complex architecture and polarization of the retina are maintained.

Overall, we have described a novel porcine model for induction of posterior vitreous detachment and demonstrated that ocriplasmin is able to induce PVD in this model. As transient hyperreflective spots and fluid were observed in this model, we further examined vitreoretinal tissues to elucidate the underlying mechanism. We did not observe significant retinal extracellular matrix rearrangement or acute inflammation after injection of ocriplasmin, but further cell-based experiments did indicate a potential transient and nontoxic effect on blood-retinal permeability. Further, 

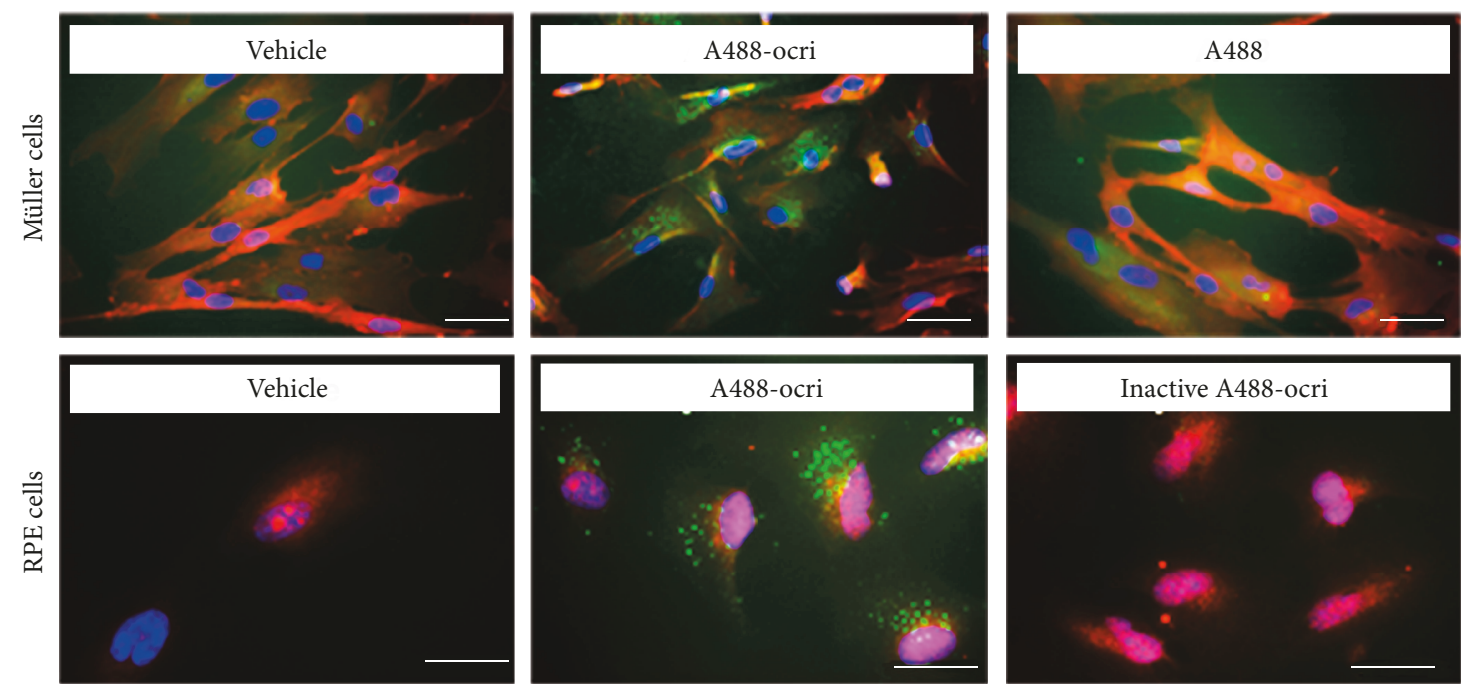

A488-ocriplasmin - CD44 Müller marker/RPE65 RPE marker - DAPI

(a)

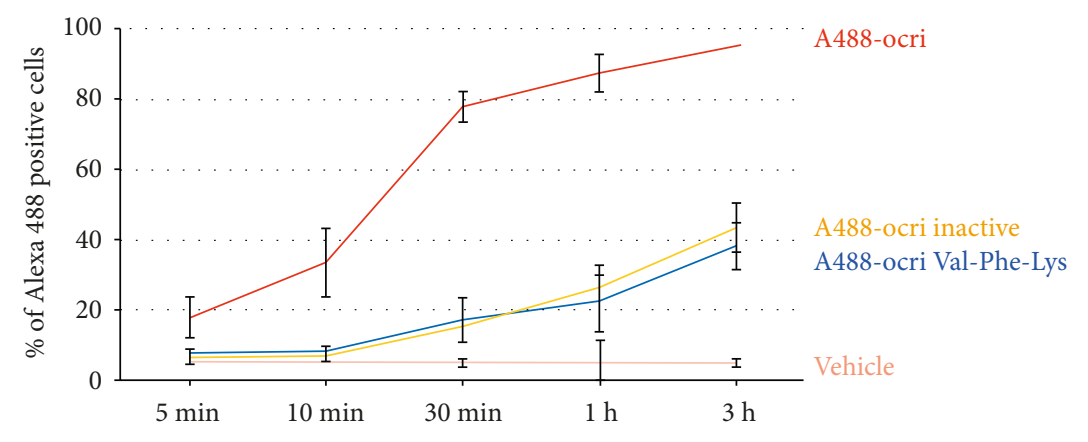

(b)
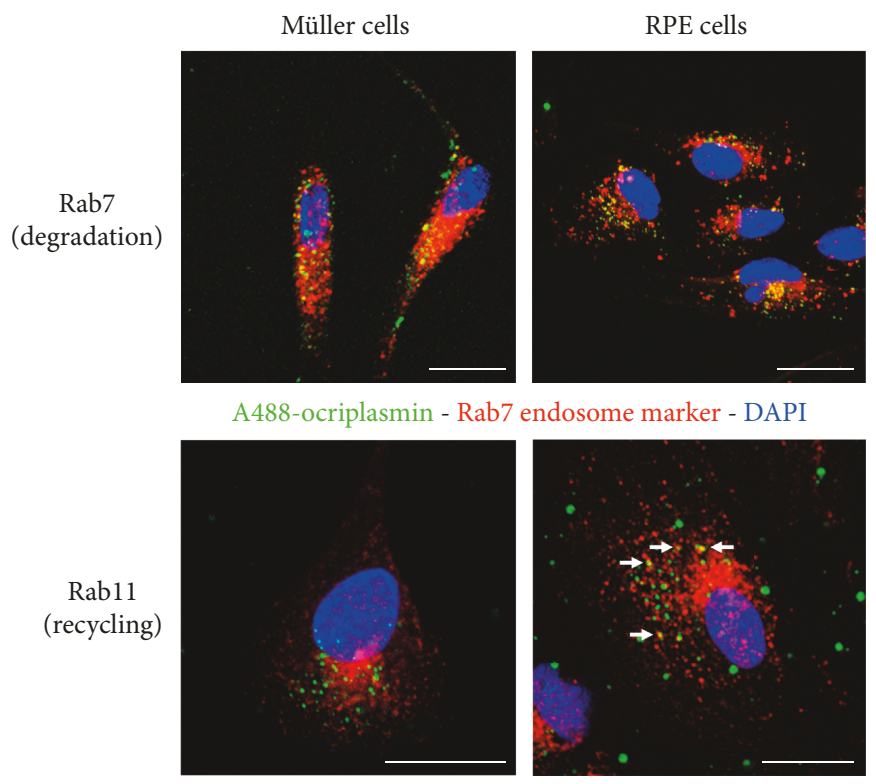

A488-ocriplasmin - Rab11 endosome marker - DAPI

(c)

FIGURE 7: Uptake of ocriplasmin by retinal cells. (a) Fluorescence microscopy of Müller and RPE cells after treatment with Alexa 488 labeled ocriplasmin. (b) Dynamics of Alexa 488 ocriplasmin uptake as studied by flow cytometry. (c) Assessment of colocalization of Alexa 488 ocriplasmin with endosomal transport vehicles by confocal microscopy. Individual channels are presented in Supplemental Figure 2. 
in vivo studies would need to be performed to ascertain whether SRF correlates with ocriplasmin-induced effects on the blood-retinal barrier.

$\begin{array}{ll}\text { Abbreviations } \\ \text { BRB: } & \text { Blood-retinal barrier } \\ \text { ECM: } & \text { Extracellular matrix } \\ \text { ERG: } & \text { Electroretinogram } \\ \text { HRS: } & \text { Hyperreflective spots } \\ \text { HRMEC: } & \text { Human retinal microvascular endothelial cells } \\ \text { IVT: } & \text { Intravitreal } \\ \text { OCT: } & \text { Optical coherence tomography } \\ \text { PVD: } & \text { Posterior vitreous detachment } \\ \text { RPE: } & \text { Retinal pigmented epithelium } \\ \text { SRF: } & \text { Subretinal fluid } \\ \text { TEER: } & \text { Transepithelial or transendothelial electrical } \\ & \text { resistance }\end{array}$

VMA/VMT: Vitreomacular adhesion/Vitreomacular traction.

\section{Conflicts of Interest}

The authors declare that there is no conflict of interest regarding the publication of this paper.

\section{Authors' Contributions}

Bart Jonckx, Michael Porcu, Aurelie Candi, Philippe Barbeaux, and Jean HM Feyen participated in the research design. Bart Jonckx, Michael Porcu, Aurelie Candi, and Isabelle Etienne conducted the experiments. Bart Jonckx, Michael Porcu, and Aurelie Candi contributed new reagents or analytic tools and performed data analysis. Bart Jonckx, Michael Porcu, Philippe Barbeaux, and Jean HM Feyen wrote or contributed to the writing of the manuscript. Bart Jonckx and Michael Porcu contributed equally to this work.

\section{Acknowledgments}

The authors would like to thank Marianne Eelen, Anne Verborg, and Tom Janssen for their technical support. This work was supported by Grant 140868 from the Agency for Innovation by Science and Technology (IWT) in Flanders, Belgium.

\section{References}

[1] J. S. Duker, P. K. Kaiser, S. Binder et al., "The international vitreomacular traction study group classification of vitreomacular adhesion, traction, and macular hole," Ophthalmology, vol. 120, no. 12, pp. 2611-2619, 2013.

[2] T. L. Jackson, E. Nicod, A. Angelis et al., "Pars plana vitrectomy for vitreomacular traction syndrome: a systematic review and metaanalysis of safety and efficacy," Retina, vol. 33, no. 10, pp. 2012-2017, 2013.

[3] J. D. Stein, D. N. Zacks, D. Grossman, H. Grabe, M. W. Johnson, and F. A. Sloan, "Adverse events after pars plana vitrectomy among Medicare beneficiaries," Archives of Ophthalmology, vol. 127, no. 12, pp. 1656-1663, 2009.
[4] P. Stalmans, M. S. Benz, A. Gandorfer et al., "Enzymatic vitreolysis with ocriplasmin for vitreomacular traction and macular holes," The New England Journal of Medicine, vol. 367, no. 7, pp. 606-615, 2012.

[5] W. Chen, W. Mo, K. Sun, X. Huang, Y. L. Zhang, and H. Y. Song, "Microplasmin degrades fibronectin and laminin at vitreoretinal interface and outer retina during enzymatic vitrectomy," Current Eye Research, vol. 34, no. 12, pp. 1057-1064, 2009.

[6] M. D. de Smet, C. Valmaggia, J. Zarranz-Ventura, and B. Willekens, "Microplasmin: ex vivo characterization of its activity in porcine vitreous," Investigative Ophthalmology \& Visual Science, vol. 50, no. 2, pp. 814-819, 2009.

[7] A. Gandorfer, M. Rohleder, C. Sethi et al., "Posterior vitreous detachment induced by microplasmin," Investigative Ophthalmology \& Visual Science, vol. 45, no. 2, pp. 641-647, 2004.

[8] M. S. Benz, K. H. Packo, V. Gonzalez et al., "A placebocontrolled trial of microplasmin intravitreous injection to facilitate posterior vitreous detachment before vitrectomy," Ophthalmology, vol. 117, no. 4, pp. 791-797, 2010.

[9] P. Stalmans, C. Delaey, M. D. de Smet, E. van Dijkman, and S. Pakola, "Intravitreal injection of microplasmin for treatment of vitreomacular adhesion: results of a prospective, randomized, sham-controlled phase II trial (the MIVI-IIT trial)," Retina, vol. 30, no. 7, pp. 1122-1127, 2010.

[10] K. Willekens, P. L. Abegao, E. Vandewalle, I. Stalmans, and P. Stalmans, "Improved efficacy of ocriplasmin for vitreomacular traction release and transient changes in optic disk morphology," Retina, vol. 35, no. 6, pp. 1135-1143, 2015.

[11] R. P. Singh, A. Li, R. Bedi et al., "Anatomical and visual outcomes following ocriplasmin treatment for symptomatic vitreomacular traction syndrome," British Journal of Ophthalmology, vol. 98, no. 3, pp. 356-360, 2014.

[12] J. A. Haller, P. Stalmans, M. S. Benz et al., "Efficacy of intravitreal ocriplasmin for treatment of vitreomacular adhesion: subgroup analyses from two randomized trials," Ophthalmology, vol. 122, no. 1, pp. 117-122, 2015.

[13] P. U. Dugel, M. J. Tolentino, L. Feiner, P. Kozma, and A. Leroy, "Results of the 2-Year Ocriplasmin for Treatment for Symptomatic Vitreomacular Adhesion Including Macular Hole (OASIS) Randomized Trial," Ophthalmology, vol. 123, no. 10, pp. 2232-2247, 2016.

[14] P. K. Kaiser, A. Kampik, B. D. Kuppermann, A. Girach, S. Rizzo, and R. C. Sergott, "Safety profile of ocriplasmin for the pharmacologic treatment of symptomatic vitreomacular adhesion/traction," Retina, vol. 35, no. 6, pp. 1111-1127, 2015.

[15] Y. Itoh, P. K. Kaiser, R. P. Singh, S. K. Srivastava, and J. P. Ehlers, "Assessment of retinal alterations after intravitreal ocriplasmin with spectral-domain optical coherence tomography," Ophthalmology, vol. 121, no. 12, pp. 25062507.e2, 2014.

[16] D. J. Warrow, M. M. Lai, A. Patel, J. Raevis, and D. M. Berinstein, "Treatment outcomes and spectral-domain optical coherence tomography findings of eyes with symptomatic vitreomacular adhesion treated with intravitreal ocriplasmin," American Journal of Ophthalmology, vol. 159, no. 1, pp. 20 30.e1, 2015

[17] C. Quezada-Ruiz, D. J. Pieramici, M. Nasir et al., "Outer retina reflectivity changes on SD-OCT after intravitreal ocriplasmin for vitreomacular traction and macular hole," Retina, vol. 35, no. 6, pp. 1144-1150, 2015. 
[18] L. Tyler, M. Singer, and D. Bell, "Long-term outcomes in patients with vitreomacular traction treated with a single intravitreal injection of ocriplasmin," Retinal Cases \& Brief Reports, vol. 11, no. 1, pp. 34-37, 2017.

[19] R. C. Quezada, D. J. Pieramici, M. Nasir, M. Rabena, and R. L. Avery, "Severe acute vision loss, dyschromatopsia, and changes in the ellipsoid zone on SD-OCT associated with intravitreal ocriplasmin injection," Retinal Cases \& Brief Reports, vol. 9, no. 2, pp. 145-148, 2015.

[20] A. Thanos, J. Hernandez-Siman, K. V. Marra, and J. G. Arroyo, "Reversible vision loss and outer retinal abnormalities after intravitreal ocriplasmin injection," Retinal Cases \& Brief Reports, vol. 8, no. 4, pp. 330-332, 2014.

[21] D. G. Birch, M. S. Benz, D. M. Miller et al., "Evaluation of full-field electroretinogram reductions after ocriplasmin treatment: Results of the OASIS Trial ERG Substudy," OASIS: Sub-Study Evaluation of Full-Field Electroretinogram in Patients with Ocripolsmin Vs Sham, AAO Las Vegas, 2017, abstract PA091 2015.

[22] R. Varma, J. A. Haller, and P. K. Kaiser, "Improvement in patient-reported visual function after ocriplasmin for vitreomacular adhesion: results of the microplasmin for intravitreous injection-traction release without surgical treatment (MIVI-TRUST) trials," JAMA Ophthalmology, vol. 133, no. 9, pp. 997-1004, 2015.

[23] T. L. Jackson, T. Verstraeten, L. Duchateau, and B. Lescrauwaet, "Visual function response to ocriplasmin for the treatment of vitreomacular traction and macular hole," Acta Ophthalmologica, 2017.

[24] D. G. Birch, M. S. Benz, D. M. Miller et al., "Evaluation of fullfield electroretinogram reductions after ocriplasmin treatment: results of the OASIS trial ERG substudy," Retina, 2017.

[25] B. C. Gilger, E. M. Abarca, J. H. Salmon, and S. Patel, "Treatment of acute posterior uveitis in a porcine model by injection of triamcinolone acetonide into the suprachoroidal space using microneedles," Investigative Ophthalmology \& Visual Science, vol. 54, no. 4, pp. 2483-2492, 2013.

[26] M. V. Kyhn, K. Warfvinge, E. Scherfig et al., "Acute retinal ischemia caused by controlled low ocular perfusion pressure in a porcine model. Electrophysiological and histological characterisation," Experimental Eye Research, vol. 88, no. 6, pp. 1100-1106, 2009.

[27] C. Scholda, R. Biowski, C. Simader, W. Hauff, S. Kaminski, and A. Lakits, "A new sponge profile for retinal detachment surgery: design and in vitro-effectiveness," Acta Ophthalmologica Scandinavica, vol. 77, no. 6, pp. 700-703, 1999.

[28] A. Garcia-Layana, J. C. Pastor, M. A. Saornil, and G. Gonzalez, "Porcine model of proliferative vitreoretinopathy with platelets," Current Eye Research, vol. 16, no. 6, pp. 556-563, 1997.

[29] Z. Y. Li, F. Wong, J. H. Chang et al., "Rhodopsin transgenic pigs as a model for human retinitis pigmentosa," Investigative Ophthalmology \& Visual Science, vol. 39, no. 5, pp. 808-819, 1998.

[30] J. W. Ross, J. P. Fernandez de Castro, J. Zhao et al., "Generation of an inbred miniature pig model of retinitis pigmentosa," Investigative Ophthalmology \& Visual Science, vol. 53, no. 1, pp. 501-507, 2012.

[31] M. L. Beauchemin, "The fine structure of the pig's retina," Albrecht Von Graefes Archive Klinische Experimentelle Ophthalmologie, vol. 190, no. 1, pp. 27-45, 1974.
[32] L. Peichl, H. Ott, and B. B. Boycott, "Alpha ganglion cells in mammalian retinae," Proceedings of the Royal Society of London - Series B: Biological Sciences, vol. 231, no. 1263, pp. 169-197, 1987.

[33] S. L. De, H. Lauwers, P. Simoens, and J. P. De Geest, "Development of the retina in the porcine fetus. A light microscopic study," Anatomia, Histologia, Embryologia: Journal of Veterinary Medicine Series C, vol. 19, no. 3, pp. 222-235, 1990.

[34] M. Garca, J. Ruiz-Ederra, H. Hernandez-Barbachano, and E. Vecino, "Topography of pig retinal ganglion cells," The Journal of Comparative Neurology, vol. 486, no. 4, pp. 361372, 2005.

[35] J. Ruiz-Ederra, M. Garcia, D. Hicks, and E. Vecino, "Comparative study of the three neurofilament subunits within pig and human retinal ganglion cells," Molecular Vision, vol. 10, pp. 83-92, 2004.

[36] J. Ruiz-Ederra, P. F. Hitchcock, and E. Vecino, “Two classes of astrocytes in the adult human and pig retina in terms of their expression of high affinity NGF receptor (TrkA)," Neuroscience Letters, vol. 337, no. 3, pp. 127-130, 2003.

[37] A. Hendrickson and D. Hicks, "Distribution and density of medium- and short-wavelength selective cones in the domestic pig retina," Experimental Eye Research, vol. 74, no. 4, pp. 435444, 2002.

[38] T. H. Mahmoud, M. C. BW 2nd, Y. Hao et al., "Lensectomy and vitrectomy decrease the rate of photoreceptor loss in rhodopsin P347L transgenic pigs," Graefe's Archive for Clinical and Experimental Ophthalmology, vol. 241, no. 4, pp. 298-308, 2003.

[39] K. Warfvinge, J. F. Kiilgaard, E. B. Lavik et al., "Retinal progenitor cell xenografts to the pig retina: morphologic integration and cytochemical differentiation," Archives of Ophthalmology, vol. 123, no. 10, pp. 1385-1393, 2005.

[40] I. Iandiev, O. Uckermann, T. Pannicke et al., "Glial cell reactivity in a porcine model of retinal detachment," Investigative Opthalmology \& Visual Science, vol. 47, no. 5, pp. 21612171, 2006.

[41] M. R. Lalonde, B. C. Chauhan, and F. Tremblay, "Retinal ganglion cell activity from the multifocal electroretinogram in pig: optic nerve section, anaesthesia and intravitreal tetrodotoxin," The Journal of Physiology, vol. 570, no. 2, pp. 325-338, 2006.

[42] N. Lassota, J. F. Kiilgaard, J. U. Prause, and la CM., "Correlation between clinical and histological features in a pig model of choroidal neovascularization," Graefe's Archive for Clinical and Experimental Ophthalmology, vol. 244, no. 3, pp. 394-398, 2006.

[43] J. Sebag, "Molecular biology of pharmacologic vitreolysis," Transactions of the American Ophthalmological Society, vol. 103, pp. 473-494, 2005.

[44] T. Laube, C. Brockmann, G. Roessler et al., "Development of surgical techniques for implantation of a wireless intraocular epiretinal retina implant in Göttingen minipigs," Graefes Archive for Clinical and Experimental Ophthalmology, vol. 250, no. 1, pp. 51-59, 2012.

[45] E. A. Atzpodien, B. Jacobsen, J. Funk et al., "Advanced clinical imaging and tissue-based biomarkers of the eye for toxicology studies in minipigs," Toxicologic Pathology, vol. 44, no. 3, pp. 398-413, 2015.

[46] M. Veurink, G. Mangioris, B. Kaufmann et al., "Development of an intravitreal peptide (BQ123) sustained release system based on poly(2-hydroxyoctanoic acid) aiming at a retinal 
vasodilator response," Journal of Ocular Pharmacology and Therapeutics, vol. 30, no. 6, pp. 517-523, 2014.

[47] A. T. Fahim, N. W. Khan, and M. W. Johnson, "Acute panretinal structural and functional abnormalities after intravitreous ocriplasmin injection," JAMA Ophthalmology, vol. 132, no. 4, pp. 484-486, 2014.

[48] M. Vezina, "Comparative ocular anatomy in commonly used laboratory animals," in Assessing Ocular Toxicology in Laboratory Animals, A. B. Weir and M. Collins, Eds., pp. 1-23, Humana Press, New York, London, 2013.

[49] G. Pinzon-Duarte, G. Daly, Y. N. Li, M. Koch, and W. J. Brunken, "Defective formation of the inner limiting membrane in laminin $\beta 2$ - and $\gamma 3$-null mice produces retinal dysplasia," Investigative Ophthalmology \& Visual Science, vol. 51, no. 3, pp. 1773-1782, 2010.

[50] R. T. Libby, C. R. Lavallee, G. W. Balkema, W. J. Brunken, and D. D. Hunter, "Disruption of laminin $\beta 2$ chain production causes alterations in morphology and function in the CNS," The Journal of Neuroscience, vol. 19, no. 21, pp. 9399-9411, 1999.

[51] S. Aisenbrey, M. Zhang, D. Bacher, J. Yee, W. J. Brunken, and D. D. Hunter, "Retinal pigment epithelial cells synthesize laminins, including laminin 5, and adhere to them through $\alpha 3$ - and $\alpha 6$-containing integrins," Investigative Ophthalmology \& Visual Science, vol. 47, no. 12, pp. 5537-5544, 2006.

[52] R. T. Libby, M. F. Champliaud, T. Claudepierre et al., "Laminin expression in adult and developing retinae: evidence of two novel CNS laminins," The Journal of Neuroscience, vol. 20, no. 17, pp. 6517-6528, 2000.

[53] L. J. Mandarino, N. Sundarraj, J. Finlayson, and H. R. Hassell, "Regulation of fibronectin and laminin synthesis by retinal capillary endothelial cells and pericytes in vitro," Experimental Eye Research, vol. 57, no. 5, pp. 609-621, 1993.

[54] P. Yang, L. Chen, R. Zwart, and A. Kijlstra, "Immune cells in the porcine retina: distribution, characterization and morphological features," Investigative Ophthalmology \& Visual Science, vol. 43, no. 5, pp. 1488-1492, 2002.

[55] E. Cohen, A. Talmon, O. Faff, A. Bacher, and Y. Ben-Shaul, "Formation of tight junctions in epithelial cells. I. Induction by proteases in a human colon carcinoma cell line," Experimental Cell Research, vol. 156, no. 1, pp. 103-116, 1985.

[56] V. A. Swystun, B. Renaux, F. Moreau et al., "Serine proteases decrease intestinal epithelial ion permeability by activation of protein kinase C $\zeta$," American Journal of Physiology. Gastrointestinal and Liver Physiology, vol. 297, no. 1, pp. G60-G70, 2009.

[57] S. Scholl, J. Kirchhof, and A. J. Augustin, "Pathophysiology of macular edema," Ophthalmologica, vol. 224, Supplement 1, pp. 8-15, 2010.

[58] R. Simo, M. Garcia-Ramirez, M. Higuera, and C. Hernandez, "Apolipoprotein A1 is overexpressed in the retina of diabetic patients," American Journal of Ophthalmology, vol. 147, no. 2, pp. 319-325.e1, 2009.

[59] X. Che, X. Q. Fan, and Z. L. Wang, "Mechanism of bloodretinal barrier breakdown induced by HIV-1 (review)," Experimental and Therapeutic Medicine, vol. 7, no. 4, pp. 768-772, 2014.

[60] S. H. Aboul Naga, M. Dithmer, G. Chitadze et al., "Intracellular pathways following uptake of bevacizumab in RPE cells," Experimental Eye Research, vol. 131, pp. 29-41, 2015.
[61] P. Heiduschka, H. Fietz, S. Hofmeister et al., "Penetration of bevacizumab through the retina after intravitreal injection in the monkey," Investigative Ophthalmology \& Visual Science, vol. 48, no. 6, pp. 2814-2823, 2007.

[62] S. N. Reid and D. B. Farber, "Glial transcytosis of a photoreceptor-secreted signaling protein, retinoschisin," Glia, vol. 49, no. 3, pp. 397-406, 2005.

[63] H. Terasaki, T. Sakamoto, M. Shirasawa et al., "Penetration of bevacizumab and ranibizumab through retinal pigment epithelial layer in vitro," Retina, vol. 35, no. 5, pp. 10071015, 2015.

[64] M. Wieffer, T. Maritzen, and V. Haucke, "SnapShot: endocytic trafficking," Cell, vol. 137, no. 2, pp. 382.e1-382.e3, 2009. 


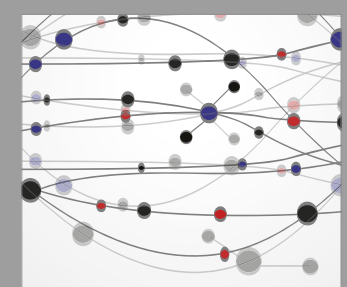

The Scientific World Journal
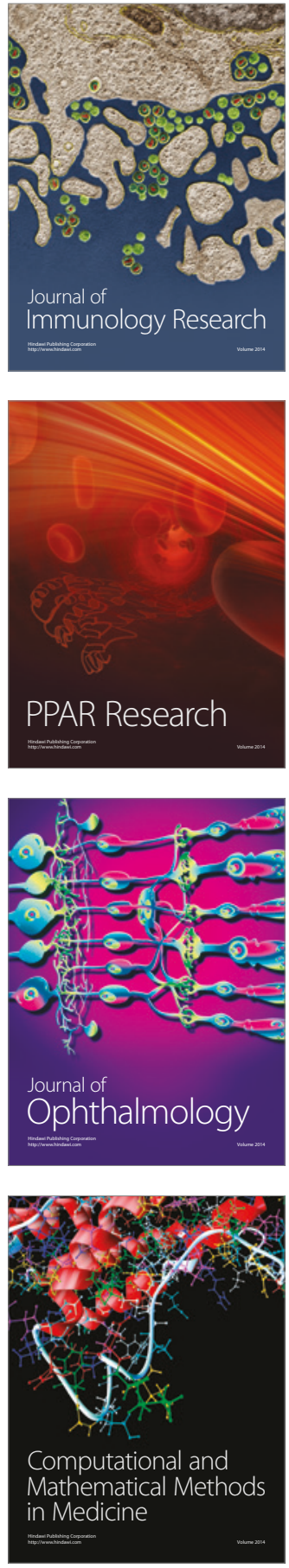

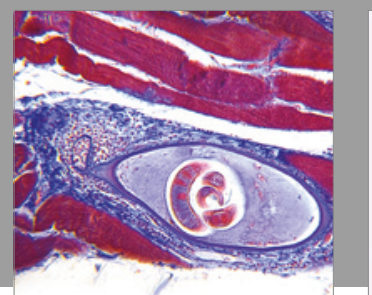

Gastroenterology Research and Practice
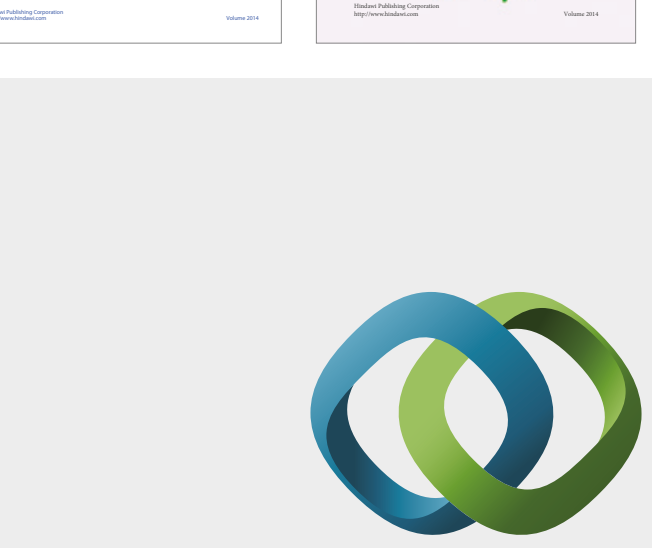

\section{Hindawi}

Submit your manuscripts at

https://www.hindawi.com
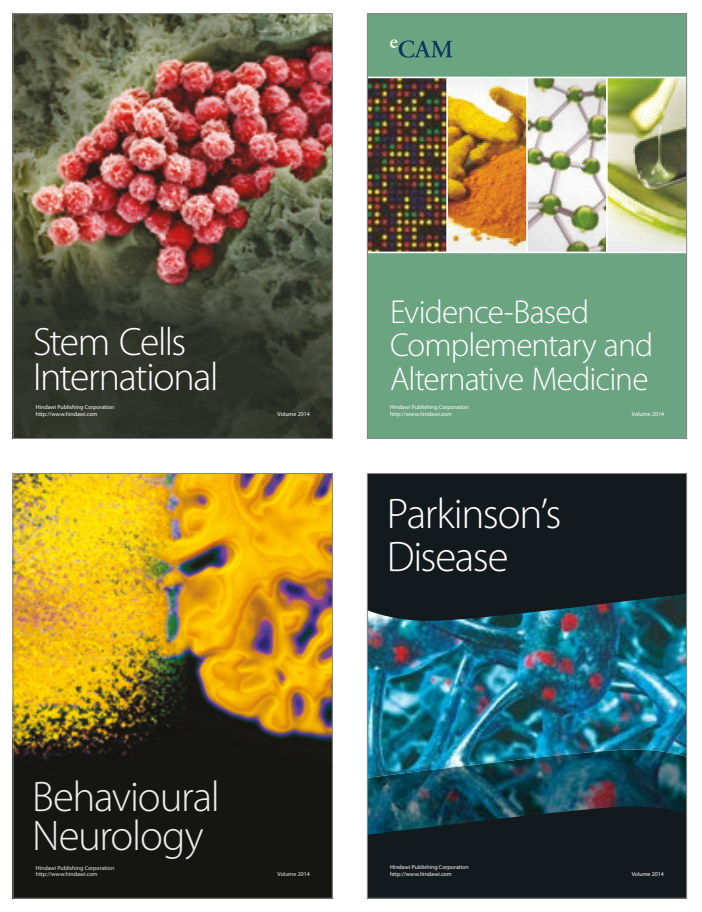
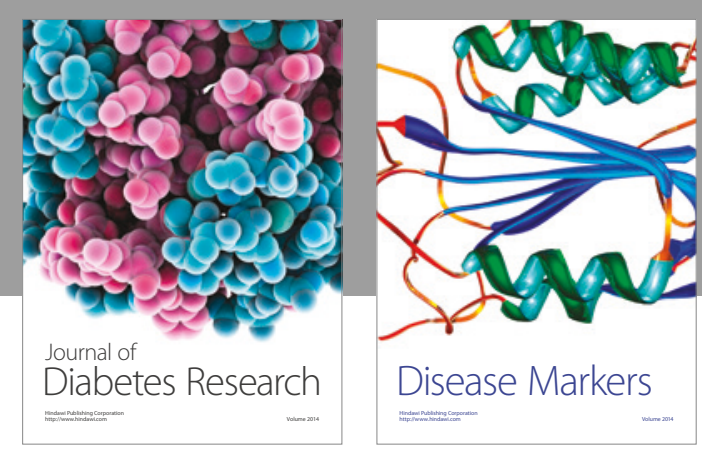

Disease Markers
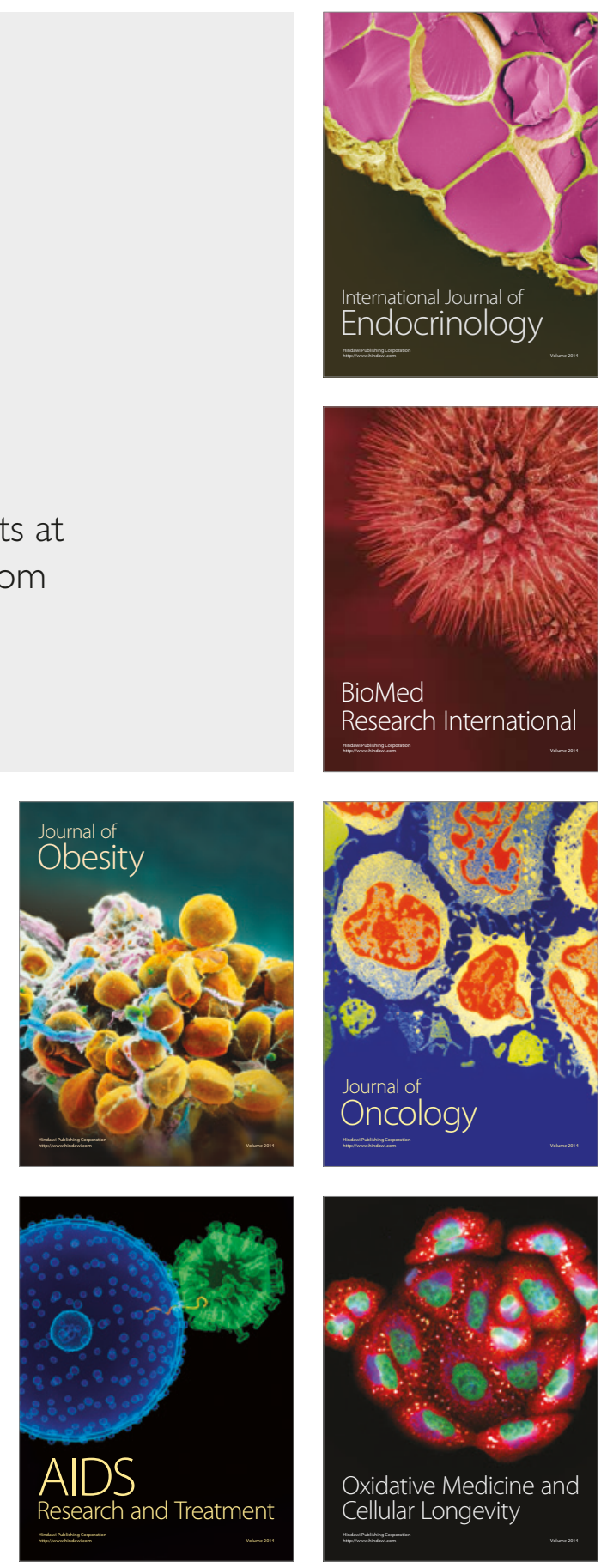